\title{
Los Sistemas de Apoyo entre Iguales (SAI) para el fomento de la convivencia en positivo, la mejora del clima de aula y la prevención de situaciones de bullying: La experiencia de Brasil y de España
}

\author{
José $\mathrm{M}^{\mathrm{a}}$ Avilés Martínez ${ }^{1}$ y Rafael Petta Daud ${ }^{2}$ \\ ${ }^{1}$ Universidad de Valladolid (España); \\ ${ }^{2}$ Universidad Estadual de Sao Paulo en Araraquara (Brasil)
}

\begin{abstract}
Se analiza la influencia de los Sistemas de Apoyo entre Iguales (SAI) en la mejora de la convivencia y el maltrato entre iguales. Se comparan dos muestras diferentes de alumnado, una con presencia de estructuras de apoyo entre iguales, los Equipos de Ayuda (Avilés, 2012), y otra sin ella tanto en escuelas secundarias de España como en Brasil. La prevalencia del bullying se obtiene tras la aplicación del Insebull (Avilés y Elices, 2007). Además se miden las conexiones y desconexiones morales de los sujetos ante dos casos de bullying, uno con víctima pasiva y otro con víctima provocativa. Se encuentran mejoras significativas en las escuelas que poseen esas estructuras respecto a la mejora del clima de convivencia y la concienciación de los sujetos implicados en las situaciones de bullying. Igualmente es significativamente mejor el grado de comunicación de las víctimas del acoso. También ocurren menos desconexiones morales en los sujetos que tienen esas estructuras de apoyo entre iguales en sus escuelas tanto frente a las víctimas pasivas como provocativas. Por ello, se hace necesario implementar y generalizar estas estructuras en el marco de los Proyectos Antibullying (Avilés, 2015) de los centros escolares.
\end{abstract}

Palabras clave: Convivencia, clima escolar, maltrato entre iguales, sistemas de apoyo entre iguales, Equipos de Ayuda.

The Peer Support Systems for the promotion of convivencia, the improvement of the classroom climate and the prevention of situations of bullying: The experience of Brazil and Spain. The influence of the Peer Support in the improvement of school climate and bullying is analyzed here. Two different student samples are compared, one with the presence of peer support structures, the Help Teams (Avilés, 2012), and another without it. Both in secondary schools in Spain and in Brazil. The prevalence of bullying is obtained after the application of Insebull (Avilés \& Elices, 2007). In addition, the moral connections and disconnections of the students are measured against two cases of bullying, one with a passive victim and another with a provocative victim. Significant improvement is found in the schools that have these structures in comparison with the improvement of the school climate and the awareness of the students involved in situations of bullying. The degree of communication of the students bullied is also significantly better. There are also fewer moral disconnections in students who have such peer support structures in their schools, when it comes to both passive and provocative victims. Therefore, it is necessary to implement and generalize these structures within the context of Antibullying Policies (Avilés, 2015) of schools.

Keywords: Coexistence, school climate, bullying, peer support, help teams.

Correspondencia: José $M^{\mathrm{a}}$ Avilés Martínez. Departamento de Psicología de la Facultad de Educación y Trabajo Social. Universidad de Valladolid. Campus Miguel Delibes. C/ Paseo de Belén nº1. C.P.: 47011. Valladolid (España). E-mail: josemaria.aviles@uva.es 
La educación en valores forma parte de los discursos programáticos de muchos sistemas educativos y de muchas escuelas, aunque su plasmación en la práctica escolar no siempre toma cuerpo a partir de programas intencionales, estructurados e insertos en el currículo de los estudiantes y en la práctica docente del profesorado.

Algunas investigaciones han puesto de manifiesto las diferencias en desarrollo moral entre los estudiantes que de forma explícita trabajan a partir de una educación en valores programada y aquellos que no lo hacen (Menin y Avilés, 2017).

Uno de los principios básicos para el trabajo en convivencia escolar es hacer partícipe y responsable de ella a quienes la comparten. En este sentido, es crucial el protagonismo concedido al alumnado en la gestión de su propia convivencia y en que las instituciones educativas posean estructuras y redes que hagan posible esa participación responsable de los estudiantes en la toma de decisiones acerca de la forma en que quieren convivir y regular sus relaciones sociales (Avilés, 2017a).

Una de las estructuras o redes que desde el mundo de los adultos se facilita en las escuelas para que el alumnado tenga un soporte desde el que posicionarse sobre decisiones morales respecto a su propia convivencia son los Sistemas de Apoyo entre Iguales (SAI), a partir de ahora SAI (Avilés, 2012 y 2017b; Avilés, Torres, y Vián, 2008; Naylor, 2010; Naylor y Cowie, 2000).

Los SAI permiten al alumnado organizarse para prestar un servicio a sus compañeros que puede ser de diversa índole, desde compañía a ayuda o a mediación. A trabajar con ellos en su desarrollo moral y a educar en valores. La diversidad de sistemas (Avilés, 2017b) permite que en función de las necesidades del grupo o de la escuela, las personas adultas que la conducen puedan brindar a sus estudiantes la modalidad de servicio en la que se puedan organizar, con el fin de solventar problemas que surgen en los grupos como conflictos, malos entendidos, aislamientos, falta de habilidades sociales de interacción, acoso o problemas coyunturales que cualquier estudiante puede sufrir a lo largo de su escolarización.

Por lo tanto, son recursos preventivos que ayudan a los estudiantes y a las escuelas a disponer de herramientas que impidan que los problemas escalen, y se hagan más grandes con el paso del tiempo, darles orientación moral sobre ellos y que sean los propios estudiantes los que cojan el timón para gestionarlos y darles una salida. Soluciones que en muchas ocasiones son mucho más efectivas porque salen y son propuestas desde el propio alumnado, que las conoce mejor que los adultos y tiene más cerca el problema.

Este trabajo provoca en los contextos educativos donde se aplica situaciones de mejora social y moral de calidad superior que en contextos en los que no se hace (Menin y Avilés, 2017), algo que hemos pretendido medir también de otra forma en esta investigación a través del estudio de los posicionamientos del alumnado ante casos de acoso, considerando el modelo de desconexiones morales de Bandura (1999) que identifica hasta ocho posibles formas de alejarse de la perspectiva moral: culpabilización 
de la víctima, lenguaje eufemístico, deshumanización y justificación moral, como formas que niegan el contenido moral en juego, además de desplazamiento de responsabilidad, difusión de responsabilidad, minimización de las consecuencias y comparación ventajosa, como formas de desconectarse sin negar el contenido moral en los casos.

\section{Marco teórico}

Los SAI son trasladados al escenario preventivo de los problemas de convivencia escolar buscando los efectos facilitadores y las ventajas, que en otros campos como el del aprendizaje proporcionaba el trabajo entre iguales (Gartner, Kohler, y Riessman, 1971; Slavin, 1995). Se constituyen como herramientas de resolución de problemas de convivencia en la tradición educativa canadiense y estadounidense (Naylor, 2010). Después se desarrollan también como apoyo a las situaciones de prevención del bullying, teniendo una extensión hoy en los centros españoles bastante amplia, aunque no generalizada.

Por el contrario, en otros contextos geográficos como Brasil, apenas se están iniciando en el tiempo, aunque con resultados esperanzadores.

Los SAI son una estrategia eminentemente preventiva que sitúa en el núcleo del grupo una estructura de sujetos entrenados que trabajan intencionalmente valores de convivencia en positivo. Su carácter proactivo provoca efectos de mejora del clima de convivencia, lo que supone una elevación del nivel de bienestar de los miembros del grupo. Se han descrito una mayor conciencia en el grupo de situaciones de acoso (Avilés et al., 2008).

Existen múltiples modalidades que permiten una versatilidad de los sistemas en función del centro educativo y de las necesidades que plantea. Se aporta ahora una muestra en la tabla 1 tomada de Avilés, Torres y Vián (2008).

En ella se señala la denominación de cada uno de ellos, la naturaleza de la relación entre quienes participan y se benefician de esos sistemas y los nombres que han llegado a adoptar en los diferentes contextos. 
Tabla 1. Modelos de Sistemas de Apoyo entre Iguales -SAI- (Avilés, Torres y Vián, 2008)

\begin{tabular}{|c|c|c|c|c|}
\hline Befriending & Peer Partnering & $\begin{array}{c}\text { Peer } \\
\text { Mediation }\end{array}$ & Peer Mentoring & Peer Counselling \\
\hline $\begin{array}{l}\text { Relación } \\
\text { colega } \\
\text { compañía } \\
\text { amistad cogida }\end{array}$ & $\begin{array}{l}\text { Relación iguales } \\
\text { Acompañamiento Estar } \\
\text { pendiente Ayuda } \\
\text { Escucha activa Apoyo } \\
\text { en decisiones } \\
\text { Comprender } \\
\text { Sentimientos y no } \\
\text { culpabilizar } \\
\text { Ayudar a buscar las } \\
\text { propias soluciones }\end{array}$ & $\begin{array}{l}\text { Dos partes- } \\
\text { Mediador } \\
\text { Encontrar } \\
\text { soluciones } \\
\text { a conflictos } \\
\text { específicos } \\
\text { Ejercicio } \\
\text { específico } \\
\text { de } \\
\text { mediación }\end{array}$ & $\begin{array}{l}\text { Mentor-Tutor } \\
\text { Orientación } \\
\text { Guía } \\
\text { Debatir, hablar, } \\
\text { discutir, } \\
\text { Reglas } \\
\text { funcionamiento } \\
\text { grupo escuela } \\
\text { Anticipación de } \\
\text { riesgos } \\
\text { Actividades } \\
\text { comunes } \\
\text { Actividades } \\
\text { sugeridas }\end{array}$ & $\begin{array}{c}\text { Relación cliente- } \\
\text { consejero } \\
\text { Escucha activa } \\
\text { Conciencia } \\
\text { social } \\
\text { Entender } \\
\text { sentimientos } \\
\text { Feedback } \\
\text { positivo } \\
\text { Ayudar a buscar } \\
\text { las propias } \\
\text { soluciones } \\
\text { Proporción } \\
\text { consejo } \\
\text { Apoyo } \\
\text { Psicológico }\end{array}$ \\
\hline $\begin{array}{l}\text { "Alumnado } \\
\text { acompañante" } \\
\text { "Equipos de } \\
\text { Acogida"“"Ayu } \\
\text { dantes de } \\
\text { recreo" }\end{array}$ & $\begin{array}{l}\text { Equipos de Ayuda } \\
\text { Alumnado Ayudante } \\
\text { Alumno Ayuda } \\
\text { "E-support" } \\
\text { "Ayudantes de recreo" }\end{array}$ & $\begin{array}{l}\text { Mediadores } \\
\text { escolares } \\
\text { Mediadores } \\
\text { en clase } \\
\text { Mediadores } \\
\text { de la } \\
\text { escuela } \\
\text { Alumnado } \\
\text { Negociador }\end{array}$ & $\begin{array}{l}\text { "Alumnado } \\
\text { Tutor" } \\
\text { "Voluntariado } \\
\text { inducido" } \\
\text { "Cybermentores } \\
\text { "“Alumnado } \\
\text { Mentor" "E- } \\
\text { support" }\end{array}$ & $\begin{array}{l}\text { "Alumnado } \\
\text { Consejero" } \\
\text { "E-support" }\end{array}$ \\
\hline
\end{tabular}

Es necesario seguir profundizando en las posibilidades educativas que todas estas modalidades tienen en nuestras escuelas con el fin de poder obtener un mayor beneficio, una mejor efectividad de los SAI en la prevención y en la resolución de los problemas de convivencia y eliminación de las situaciones de violencia escolar. Es cierto que las escuelas tienen todavía un déficit formativo importante en los procedimientos más adecuados para hacer la aplicación, y sobre todo la formación, de cada uno de estos sistemas, especialmente en contextos educativos menos privilegiados como son los de Brasil. Aun así, hemos de seguir mostrando las posibilidades y las contraindicaciones que cada uno de los formatos tienen respecto a los problemas de convivencia a resolver. No resulta útil utilizar sistemas de mediación frente a problemas de acoso con fuerte desequilibrio entre las partes o sistemas de mentoría ante problemas académicos. Podríamos pensar que a los problemas de acoso, con carácter general, se adaptan mejor sistemas de ayuda y para intentar resolver los problemas de retraso académico son más efectivos los sistemas de tutoría. En definitiva, se hace necesaria una formación que permita facilitar en los maestros y maestras que gestionan las escuelas las herramientas didácticas que les permitan aplicar de forma más idónea y efectiva cada uno de estos sistemas.

Uno de los SAI más generalizados son los Equipos de Ayuda (Avilés, 2012, 2017a y b; Avilés et al., 2008). Se trata de un sistema de los más sencillos, 
al no exigir al alumnado habilidades demasiado sofisticadas para ejercerlo. Los constituyen tres o cuatro alumnos del grupo, que son elegidos por sus compañeros para formar un Equipo que a lo largo del curso va a estar en disposición de ayudarles en las problemáticas que se les planteen. Previamente, recibirán la formación necesaria para realizar esa misión. El trabajo fundamental de quienes participan en este modelo es la de prestar ayuda a quienes lo necesiten o lo soliciten, empleando un catálogo de técnicas y habilidades que les permitan a los ayudados, ser capaces de encontrar un camino para resolver sus dificultades o problemas (Avilés, 2017b).

Este trabajo genera un ambiente en el grupo clase donde se promocionan valores morales de colaboración, estar pendiente de las necesidades de los otros y de prestación de ayuda a quienes la necesitan. Este compromiso mejora las relaciones interpersonales de los miembros promocionando posicionamientos morales más avanzados entre quienes los practican y en quienes los contemplan, ya que el discurso imperante hace referencia a la ayuda y a su prestación. Por eso, se hace necesario también precisar si los posicionamientos morales del alumnado en estos contextos, resulta diferente a los de otros, al participar de una cultura compartida de valores que apunte en esa dirección. Nuestras preguntas de investigación se centran en conocer si un sistema como este, activado en la mayoría de los grupos del centro, varía la percepción del alumnado frente a las situaciones de bullying respecto a grupos donde este sistema no esté presente. Por otra parte, también se ha pretendido analizar si se produce una mejora en sus decantaciones morales frente a esas situaciones de acoso, en función de la presencia o no de esta cultura de ayuda en cada contexto.

\section{MÉTODO}

Se mide la influencia de los Equipos de Ayuda en España y en Brasil respecto de dos situaciones, el bullying en la convivencia de los grupos y el posicionamiento de los sujetos ante estos escenarios. En definitiva, una plasmación práctica de adhesión a los valores que se poseen (Menin y Avilés, 2017).

\section{Sujetos}

La muestra de alumnado participante por países aparece en la tabla 2.

Tabla 2. Distribución de la muestra de alumnado por países

\begin{tabular}{lccc}
\hline & Brasil & España & Total \\
\hline Sin Equipos de Ayuda & $130(20.7 \%)$ & $195(44.6 \%)$ & 325 \\
\hline Con Equipos de Ayuda & $500(79.3 \%)$ & $243(55.4 \%)$ & 743 \\
\hline Total & $630(100 \%)$ & $438(100 \%)$ & 1068 \\
\hline
\end{tabular}


En España, conforme se indica en la tabla 3, el alumnado participa en dos grupos, uno de 243 chicos y chicas que desarrollan un Programa de Equipos de Ayuda y otro de 195 chicos y chicas que no participan, de entre 11 y 14 años de Educación Secundaria Obligatoria de centros públicos de Castilla y León. Se constató la igualdad de los grupos comparando sus varianzas, contrastando sus medias y mediante la prueba de Levene.

Tabla 3. Distribución por agrupamiento y sexo de la muestra española y brasileña

\begin{tabular}{lccccccc}
\hline \multicolumn{7}{c}{ España } & \multicolumn{2}{c}{ Brasil } & & \\
\hline & Chicos & Chicas & Chicos & Chicas & Total España & Total Brasil & Total \\
\hline $\begin{array}{l}\text { Con Equipos } \\
\text { de Ayuda }\end{array}$ & $123(51.4 \%)$ & $120(48.6 \%)$ & $259(51.8 \%)$ & $241(48.2 \%)$ & $243(55.02 \%)$ & $500(52.2 \%)$ & $743(69.57)$ \\
\hline $\begin{array}{l}\text { Sin Equipos } \\
\text { de Ayuda }\end{array}$ & $107(54.9 \%)$ & $88(45.1 \%)$ & $74(56.92 \%)$ & $56(43.08 \%)$ & $195(44.98 \%)$ & $130(47.8 \%)$ & $325(30.43)$ \\
\hline Total & $230(52.52 \%)$ & $208(47.48 \%)$ & $333(52.86 \%)$ & $297(47.14 \%)$ & $438(100 \%)$ & $630(100 \%)$ & $1068(100 \%)$ \\
\hline
\end{tabular}

En Brasil, la muestra se reparte en dos grupos, uno de 500; chicos y chicas de centros que tienen Equipos de Ayuda y otro de 130 chicos y chicas de centros que no los tienen, todos de centros públicos y privados del Estado de Sao Paulo.

\section{Instrumentos}

Para la medición de la prevalencia del bullying en los grupos de ambos países se utilizó el instrumento Insebull (Avilés y Elices, 2007) que fue validado mediante el método de componentes principales no lineales y por juicio de expertos (Alpha=.9634). Su fiabilidad obtuvo un Alpha de Crombach de .8678.

Para la valoración del posicionamiento moral del alumnado se utilizó un cuestionario que fue adaptado a la realidad de cada país. Validado en investigaciones anteriores, presenta dos situaciones hipotéticas de bullying, una de una víctima 'típica' y otra de una víctima 'provocadora', seguidas de 14 alternativas que las explican. De las alternativas, ocho responden a las categorías de desconexión moral descritas por Bandura (1991, 1999) y seis a las de conexión moral, o sea, en las que se reconoce la falta de respeto en las acciones de maltrato que se ocasiona a los personajes principales de esas situaciones. investigación.

En ambos países se guardaron los procedimientos éticos exigibles a este tipo de

\section{Procedimiento}

En España, quienes participaron en la evaluación fueron alumnado de los Equipos de Ayuda y los receptores del servicio. Los Equipos de Ayuda y el profesorado responsable recibieron la formación necesaria para desarrollar el Programa. El alumnado participó en un curso de formación de dos días en que se desarrollaron contenidos sobre convivencia, resolución de conflictos, comunicación, empatía, asertividad y trabajo en 
equipo. El profesorado se preparó previamente para llevar a cabo este curso de formación. Se elaboraron materiales específicos para desarrollar la formación. En Brasil el alumnado perteneciente a los Equipos de Ayuda recibió un curso de formación antes de su implantación.

Los menores fueron debidamente autorizados por sus padres o tutores responsables y orientados para cumplimentar los dos instrumentos, el Insebull primero y el cuestionario de posicionamiento moral después. En este último instrumento, el alumnado fue categorizado en cuatro niveles de desempeño moral, establecidos entre el nivel 1 (el más bajo, en el cual están quienes solo se adhieren a alternativas de desconexión moral) y el nivel 4 (el más alto, que acoge a los sujetos que eligen las alternativas de conexión moral). El alumnado elegía entre las catorce alternativas que se le ofrecían respecto a dos casos de bullying. Uno prototípico de una víctima pasiva y otro de una víctima provocativa. Cada una de las alternativas a elegir denotaban una posición de conexión o desconexión moral.

\section{RESULTADOS}

Se muestran resultados de los dos estudios de la investigación.

\section{Estudio I}

En ambos países los índices de prevalencia del bullying encontrados no son significativamente diferentes cuando los que responden están involucrados o bien como agresores o bien como víctimas. Sin embargo, son significativamente diferentes cuando se valoran como espectadores y entre los dos países, como se observa en la tabla 4.

Tabla 4. Respuestas al ítem 26 del cuestionario Insebull (Avilés y Elices, 2007)

\begin{tabular}{|c|c|c|c|c|c|c|c|}
\hline \multirow{3}{*}{$\begin{array}{l}\text { ¿Con qué frecuencia han } \\
\text { ocurrido intimidaciones } \\
\text { en tu Centro? }\end{array}$} & \multicolumn{4}{|c|}{ Equipos de Ayuda } & \multirow{3}{*}{$\begin{array}{l}\text { Total } \\
\text { Valor-p } \\
\text { España }\end{array}$} & \multirow{3}{*}{$\begin{array}{l}\text { Total } \\
\text { Valor-p } \\
\text { Brasil }\end{array}$} & \multirow{3}{*}{$\begin{array}{l}\text { Valor- } \\
\mathrm{p}\end{array}$} \\
\hline & \multicolumn{2}{|c|}{ España } & \multicolumn{2}{|c|}{ Brasil } & & & \\
\hline & No & Sí & No & $\mathrm{Si}$ & & & \\
\hline Nunca & $32(17.3 \%)$ & $89(37.9 \%)$ & $130(43.05 \%)$ & $156(34.44 \%)$ & 121 & 286 & \\
\hline Menos de cinco veces & $75(40.5 \%)$ & $89(37.9 \%)$ & $52(17.22 \%)$ & $121(26.71 \%)$ & 164 & 173 & \\
\hline Entre cinco y diez veces & $46(24.9 \%)$ & $23(9.8 \%)$ & $28(9.27 \%)$ & $88(19.43 \%)$ & 69 & 116 & \\
\hline Entre diez y veinte veces & $7(3.8 \%)$ & $8(3.4 \%)$ & $6(1.99 \%)$ & $25(5.52 \%)$ & 15 & 31 & $<0.01$ \\
\hline Más de veinte veces & $6(3.2 \%)$ & $12(5.1 \%)$ & $12(3.97 \%)$ & $16(3.53 \%)$ & 18 & 28 & \\
\hline Todos los días & $19(10.3 \%)$ & $14(6 \%)$ & $74(24.5 \%)$ & $47(10.38 \%)$ & 33 & 121 & \\
\hline Total & 185 & 235 & 302 & 453 & 420 & 755 & \\
\hline
\end{tabular}

La frecuencia de las intimidaciones son significativamente diferentes $(p<0.01)$ entre los centros españoles y brasileños, tanto en contextos con Equipos de Ayuda como en aquellos que no los tienen. En los brasileños que no tienen Equipos de Ayuda, prácticamente se dobla la frecuencia de intimidación que sucede todos los días $(24.5 \%)$ frente a lo que ocurre en los españoles $(10.27 \%)$. Respeto a los centros que sí disponen de Equipos de Ayuda también se encuentran diferencias $(p>0.01)$ entre escuelas brasileñas 
y españolas, ya que la frecuencia de intimidaciones (nunca -34.44\%- en Brasil y -37.89\%en España) suceden más en Brasil que en España (todos los días -10.38\%- en Brasil frente al $-5.96 \%$ - en España).

Otros resultados entre centros con y sin Equipos de Ayuda se refieren a contenidos como la gestión emocional de las situaciones $(p>0.02)$ y las atribuciones causales que hacen las víctimas $(p>0.02)$, la respuesta que reciben los agresores $(p>0.01)$, la imagen que percibe el grupo de ellos $(p>0.01)$ y la atribución causal que hacen de los hechos $(p>0.01)$, o las relacionadas con la comunicación de los hechos a los adultos $(p>0.01)$ y la intervención de éstos en las situaciones de bullying para pararlas $(p>0.01)$. En estas variables los centros donde hay Equipos de Ayuda muestran datos significativamente mejores que los que no los tienen, en ambos países.

Por su parte, en España los centros con Equipos de Ayuda estimulan más la respuesta que los espectadores dan a los casos de acoso $(p>0.01)$ y se decantan más claramente en defensa de las víctimas $(p>0.01)$ que aquellos que no disponen de estos sistemas de apoyo. Sin embargo, en Brasil destacan por hacer propuestas de salida a las situaciones $(p>0.01)$ y reactivar a las víctimas en las respuestas de defensa que emiten $(p>0.01)$.

\section{Estudio II}

En general, se observa que el alumnado que convive con los Equipos de Ayuda, tanto español como brasileño, manifiesta un menor índice de desconexiones morales que el alumnado sin Equipos de Ayuda. Así, el 67.4\% del alumnado perteneciente a la muestra brasileña que no tiene cerca Equipos de Ayuda señalan la alternativa correspondiente a la desconexión moral denominada 'lenguaje eufemístico' correspondiente a la primera historia que se contesta, que es la que corresponde con la víctima pasiva, frente al $41.82 \%$ de colegas con estructuras de Equipos de Ayuda en sus centros $(p<0.01)$. En el caso de los españoles, el 5.68\% del alumnado que no está en centros con Equipos de Ayuda opta por la desconexión moral denominada 'desplazamiento de responsabilidad' en la historia correspondiente a la víctima provocativa, frente al $0.78 \%$ entre el alumnado con Equipos de Ayuda en sus centros $(p<0.03)$.

Entre países, son significativamente más las formas de desconexión moral en Brasil que en España, tanto entre el alumnado de centros con Equipos de Ayuda como sin ellos. En ambos tipos de centros, las diferencias se centran en los niveles más bajos (Nivel 1), en los que prevalecen las formas de desconexión moral, como se observa en la tabla 5. También sucede en el nivel 3 (sociocéntrico), en general, tanto en centros con Equipos de Ayuda, como en centros sin Equipos de Ayuda, y frente a los dos tipos de víctima. 
Tabla 5. Diferencias en desconexión moral entre el alumnado en los casos de bullying que enfrentan

\begin{tabular}{|c|c|c|c|c|}
\hline Nivel 1 & Brasil $(n=461)$ & España $(n=459)$ & Total & Valor- $p^{*}$ \\
\hline \multicolumn{5}{|c|}{ Con Equipos de Ayuda } \\
\hline Víctima Pasiva & $12(9.23 \%)$ & $6(5.83 \%)$ & $18(7.73 \%)$ & 0.01 \\
\hline \multicolumn{5}{|c|}{ Sin Equipos de Ayuda } \\
\hline Víctima Provocativa & $26(5.62 \%)$ & $11(2.04 \%)$ & $37(4.01 \%)$ & 0.01 \\
\hline
\end{tabular}

La modalidad de víctima, por tanto, despierta explicaciones diferentes entre el alumnado. En el caso de la provocativa, el alumnado con Equipos de Ayuda sitúa sus posiciones en estadíos significativamente de más desarrollo moral (F3-sociocéntrica- y F4-moral) que el alumnado que no posee esos equipos.

Además, respecto a la elección de las alternativas que se ofrecen frente a los casos, se constatan marcadas diferencias $(p<0.01)$ entre el alumnado que tienen Equipos de Ayuda y el que no. En el caso de la víctima pasiva, los centros que tiene Equipos de Ayuda eligen alternativas significativamente menos desconectadas moralmente $(p<0.01)$ que en los otros centros. Por ejemplo, eligen como explicación de lo que pasa 'la culpabilización de la víctima' un $10 \%$ frente a un $40 \%$ en los centros sin Equipos de Ayuda; la 'minimización de las consecuencias' un $6.5 \%$ frente a un $24.6 \%$; o la 'deshumanización de la víctima' un $9.5 \%$ frente a un $23.8 \%$. Datos similares aparecen también frente al caso de la víctima provocativa.

\section{DISCUSIÓN}

\section{Estudio I}

Los resultados encontrados en esta investigación refuerzan otros anteriores (Avilés, et al., 2008; Cowie y Wallace, 2000; Menesini et al., 2003). Sugieren que, cuando se afrontan situaciones de intimidación, donde la violencia ocurre entre iguales, los denominados Equipos de Ayuda, se convierten en una herramienta valiosa para la lucha o para la prevención de estas situaciones, para mejorar el ambiente de concienciación contra el bullying y como modelos donde se miran los iguales para medir sus posicionamientos. Este sistema de apoyo, basado en el modelo español (Avilés, 2012) de los Equipos de Ayuda, actualmente se viene implantando también en algunas escuelas brasileñas, lo que nos permite realizar comparaciones en contextos tan diferentes. La mayor percepción de prevalencia del bullying entre el alumnado brasileño frente al español, tanto con y sin Equipos de Ayuda, es un exponente de ese contraste. Diferencias que se marcan también en otras investigaciones relacionadas con la adhesión a valores (Menin y Avilés, 2017) como el de la convivencia democrática. Sin embargo, realidades educativas tan diferentes permiten constatar la mejora común que sistemas como los Equipos de Ayuda proporcionan en la prevención de la violencia entre iguales y la gestión del bullying, tanto en víctimas y testigos como en agresores (Avilés et al., 2008; Menesini et al., 2003). 
En víctimas, es la posibilidad de comunicar lo que les sucede o tener interlocutores favorables para trasmitirlo, lo que supone un salto cualitativo. A diferencia de los centros que no disponen de estos sistemas, los Equipos de Ayuda favorecen en las víctimas un punto de conexión con lo que les pasa y una ayuda para encontrar vías de solución posible, algo que se constata más en el contexto brasileño que en el español. Sin duda, son los iguales dispuestos a ayudar quienes están en una mejor posición para echar una mano cuando hace falta (Cowie y Oztug, 2008).

Quienes agreden también se ven afectados por la presencia de los Equipos de Ayuda, pues su actuación y el ambiente que crea en el grupo la disposición por la ayuda, les obliga a percibir, evaluar y tomar conciencia de la calidad de los actos que realizan (Avilés et al., 2008). Este movimiento es favorable para que en los centros construyan iniciativas para gestionar de forma diferente los modelos disciplinarios y normativos en su seno; articular actuaciones que generen compromisos para la resolución del bullying (Aviles, 2006; Pikas, 1989; Rigby, 1996); e incluso, valorar la efectividad de otras actuaciones específicas encaminadas a combatir el maltrato, aprovechando el clima de concienciación que genera el Programa.

También los testigos se ven afectados, pues algunos forman parte de los Equipos de Ayuda. A los testigos se les obliga a reflexionar sobre todas las formas de maltrato, incluso sobre las que pueden quedar más ocultas o desapercibidas, y que no por eso son menos dañinas. Es el caso del maltrato social, la exclusión o el aislamiento por inacción.

\section{Estudio II}

Entre el alumnado, la ausencia de Equipos de Ayuda en los centros se manifiesta como un factor importante para el posicionamiento moral frente a casos de bullying y favorece que los sujetos se sitúen preferentemente en opciones de desconexión moral, especialmente en el contexto brasileño y frente a los casos de víctimas provocativas, con las que es más difícil gestionar la convivencia en el grupo (Avilés, 2006). Considerando el hecho de que la desconexión moral se produce en la medida en que los sujetos son poco capaces de reconocer el contenido moral que sucede cuando hay una falta de respeto como ocurre en el bullying, los resultados obtenidos nos permiten afirmar que los Equipos de Ayuda y su influencia y acciones se configuran como una variable importante para que los sujetos que eventualmente puedan estar implicados, directa o indirectamente, en una situación de bullying tengan razones para rechazar este fenómeno por ser algo violento y dañino y, consecuentemente, estar en condiciones para hacer juicios morales adecuados y más conectados, con el fin de actuar buscando su superación.

Parece claro que un contexto educativo y de prevención de la violencia en red entre iguales en el que predomina el fomento de valores morales (Menin y Avilés, 2017), tomando forma en una estructura como los Equipos de Ayuda, permite en el alumnado 
tener presentes modelos de juicio moral frente al bullying de mayor conexión moral, disminuyendo opciones como la culpabilización de las víctimas, la minimización de las consecuencias de esos actos o la asignación de perfiles deshumanizantes en la víctima como soportes cognitivos de la exculpación y para hacer más soportable el maltrato.

Será necesario indagar con futuras investigaciones las diferencias en el nivel de posicionamiento moral que provoca en el alumnado el perfil de víctima que tienen frente a ellos. Cómo se puede minimizar la provocación en la víctima para que no oculte su propia condición de víctima ante los ojos de quienes conviven con ella. En definitiva, cómo se puede visualizar más esa necesidad en la propia interacción. Y porqué la inacción de la víctima pasiva no despierta más acciones de ayuda entre quienes se desconectan moralmente, a pesar de la visualización que hace de su condición.

\section{CONCLUSIONES}

Podemos decir que la implantación de Sistemas de Ayuda ha contribuido a crear un clima de concienciación muy significativo contra el maltrato y a favor de la convivencia (Avilés, 2017a). En la comunidad educativa donde se habla de los problemas de convivencia y de bullying, permite que se haga presente un mensaje institucional contra el abuso y se respalden oficialmente las estructuras (los Equipos de Ayuda) que hacen posible la intervención.

En definitiva, los datos de la investigación referidos tanto a España como a Brasil, parecen también indicar que los Equipos de Ayuda son una buena herramienta para mejorar la convivencia en la escuela y para prevenir situaciones de bullying. Incluso, para hacer decantar las posiciones morales entre el alumnado que se beneficia de ellos en los entornos de convivencia de sus centros, hacia perspectivas más conectadas moralmente. Modelos de actuación moral que tienen el aval del aprendizaje entre iguales y el modelado y tirón del liderazgo positivo, que permite hacer visibles y patentes los valores de ciudadanía que se quieren promover. Sin embargo, además se hace necesario tomar otras medidas específicas para reducir la prevalencia del acoso en las escuelas. Estas medidas necesitan organizarse en torno a lo que llamamos un Proyecto Antibullying (Avilés, 2015), donde participen todos los miembros de la comunidad educativa, incluido el alumnado, que permitan conducir y vertebrar las actuaciones que contra el maltrato se organicen en su seno.

La experiencia con el alumnado nos indica que la posibilidad de darles la voz y el tiempo como protagonistas de sus propias acciones y un papel importante en la gestión de los casos de bullying, les ayuda a experimentar una autonomía en las escuelas, que les permite empoderarse para ser más libres en el futuro. Conviene recordar que el papel de protagonismo del alumnado en los sistemas educativos español y brasileño es muy desigual. En el caso español, existe una trayectoria implantada en los centros y cierta 
variedad en la tipología de sistemas (Avilés, 2017b), mientras que en el sistema educativo brasileño carece de historia, habiendo quedado relegado el alumnado a una función receptora de órdenes y un cumplimiento de las decisiones de la autoridad. Apenas ahora se están iniciando experiencias que ayudan a difundir el modelo en el Estado de Sao Paulo y en otras ciudades de Brasil. Un contraste así, hace de los resultados de esta investigación un espaldarazo importante para el respaldo, la implantación y la posible generalización en las escuelas de sistemas como los Equipos de Ayuda.

En el caso del alumnado de la investigación actual, si al enfrentar a los estudiantes de centros con Equipos de Ayuda a dos situaciones de violencia conocida, ellos eligen opciones que suponen más conexión moral y menos desconexión ante esos casos, podemos considerar una estrecha relación entre esas elecciones y los valores que los Equipos de Ayuda proponen.

Es posible reconocer que la implantación de los Equipos de Ayuda ofrece a las escuelas que los adoptan y a los grupos de convivencia que los tienen, un cambio en el ambiente de confianza entre pares y en la presencia y gestión de los valores morales que manejan y que suponen las situaciones de bullying, lo que son oportunidades plausibles para la reflexión y el crecimiento personal. Objetivos que conforman un modelo de convivencia positiva (Avilés, 2017a) como el que trabajamos.

\section{REFERENCIAS}

Avilés, J.M. (2006). Bullying: el maltrato entre iguales. Agresores, víctimas y testigos en la escuela. Salamanca: Amarú.

Avilés, J.M (2012). Manual contra el bullying. Guía para el profesorado. Lima: Libro Amigo.

Avilés, J.M. (2015). Proyecto Antibullying. Prevención del bullying y el cyberbullying en la comunidad educativa. Madrid: CEPE.

Avilés, J.M. (2017a). Convivencia: a strategy that prevents school bullying and promotes emotional health and well-being. En H. Cowie and A-M Myers (Eds.) School Bullying and Mental Health: Risks, Intervention and Prevention. London: Routledge.

Avilés, J.M. (2017, b). Los Sistemas de Apoyo entre Iguales (SAI) y su contribución a la convivencia escolar. Innovación Educativa, (27), 5-18.

Avilés, J.M., Torres, N., y Vian, M.V. (2008). Equipos de ayuda, maltrato entre iguales y convivencia escolar. Electronic Journal of Research in Educational Psychology, 6(3), 357-376.

Bandura, A. (1999). Moral disengagement in the perpetration of inhumanities. Personality and Social Psychology Review, 3(3), 193-209. http://dx.doi.org/ 10.1207/s15327957pspr0303_3

Cowie, H., y Wallace, P. (2000). Peer support in action. London: Sage.

Cowie, H., y Oztug, O. (2008). Pupils' perceptions of safety at school. Pastoral Care in Education, 26(2), 59-67. doi: 10.1080/02643940802062501

Gartner, A., Kohler, M.C., y Riessman, F. (1971). Children teach children. Nueva York: Harper Row.

Kohlberg, L. (1992). Psicología del desarrollo moral. Bilbao: Desclée de Brouwer. 
Menesini, E., Codecasa, E., Benelli, B., y Cowie, H. (2003). Enhancing children's responsibility to take action against bullying: Evaluation of a befriending intervention in Italian middle schools. Aggressive Behavior, 29, 10-14.

Menin, M.S., Avilés, J.M., y Tavares, M. (2017). Adhesión a valores de convivencia en niños y adolescentes españoles y brasileños. Estudio preliminar de una investigación transcultural. Comunicación en el I Congreso Internacional de Psicología, Salud y Educación. Oviedo: CIPSE.

Menin, M.S. y Avilés, J.M. (2017). Adhesión a valores de convivencia en niños y adolescentes españoles y brasileños. Estudio preliminar de una investigación transcultural. En J.C. Núñez, M.C. Pérez-Fuentes, M.M. Molero, J.J. Gázquez, A. Martos, A.B. Barragán y M.M. Simón (Eds.) Temas actuales de investigación en las áreas de la Salud y la Educación, pp.419-426. Almería: SCINFOPER.

Naylor, P. (2010). Anti-bullying peer support systems in school: What are they and dothey work? Ponencia impartida en el II International Congress of Community Life at School. Almería: Universidad de Almeria.

Naylor, P., y Cowie, H. (2000). The role of peer support systems in education for citizenship. The School Field, 11(1-2), 131-142.

Pikas, A. (1989).The common concern method for the treatment of mobbing. En Roland, E. y Munthe, E. (Eds.). Bullying: An international perspective (pp. 91-105). London: David Fulton.

Rigby, K. (1996). Bullying in schools. And what to do about it. London: Jessica Kingsley.

Slavin, R.E. (1995). Cooperative learning. Theory, research and practice. Massachusetts: Allyn y Bacon.

Recibido: 20 de noviembre de 2017

Recepción Modificaciones: 11 de enero de 2018

Aceptado: 17 de enero de 2018 